\title{
La evolución de la investigación para la salud redefinirá las agendas nacionales de salud*
}

\author{
Carissa Etienne ${ }^{1}$, Kamran Abbasi ${ }^{2}$ y Luis Gabriel Cuervo ${ }^{3}$
}

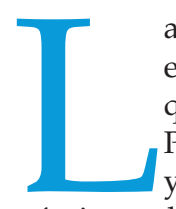

a investigación para la salud es el eje de los sistemas de salud que son eficaces y eficientes. Para mejorar la salud pública y comprender los aspectos económicos y la mecánica de los sistemas de salud, se requiere una amplia gama de métodos de investigación. En los últimos decenios, con el rápido incremento en el acceso al conocimiento científico, también han aumentado las expectativas de que ese conocimiento se aplique y se traduzca en una mejor salud. Hoy en día, la sociedad espera que las decisiones estén fundamentadas en el mejor conocimiento disponible, pero todavía no se organizan ni se gestionan de manera adecuada las iniciativas de investigación. En consecuencia, gran parte de la investigación que se realiza se desperdicia o tiene un valor limitado. Los patrocinadores de las investigaciones no ven que su inversión incida en el desarrollo, y es posible que no vean siquiera algún tipo de efecto en las iniciativas de prevención o las políticas de salud..$^{1-5}$

En el 2009, los Estados Miembros de la Organización Panamericana de la Salud (OPS) aprobaron la Politica de investigación para la salud de la Región de las Américas, la primera política regional de este tipo en la Organización Mundial de la Salud (OMS). ${ }^{6}$ Esta política fue elaborada en consonancia con la Estrategia de la OMS sobre investigaciones en pro de la salud y la complementa. ${ }^{7}$ En esta serie especial de la OPS y The BMJ (www.bmj.com/health -research-americas) se presentan algunas perspectivas y apreciaciones sobre lo que

\footnotetext{
* Traducción oficial al español efectuada por la Organización Panamericana de la Salud. En caso de discrepancia, prevalecerá la versión original en inglés.
}

ha ocurrido tras la aplicación de la política de investigación de la OPS, así como los retos que han surgido. Analizamos lo que nos depara el futuro en vista de la adopción de la Agenda 2030 para el Desarrollo Sostenible de las Naciones Unidas y sus Objetivos de Desarrollo Sostenible, del llamamiento cada vez mayor a favor de la cobertura universal de salud y el fortalecimiento de los sistemas de salud, y de la aprobación en mayo del 2018 del 13. ${ }^{\circ}$ Programa General de Trabajo de la OMS para el período 2019-2023. ${ }^{8}$

Tras la introducción de la política regional, la mayor parte delos Estados Miembros de la OPS elaboraron políticas nacionales de investigación para la salud. Algunas de ellas ya no están vigentes o están siendo actualizadas. A diciembre del 2017, 16 países habían informado que tenían una política nacional de investigación para la salud, y 18 países de la Comunidad del Caribe (CARICOM) respaldaban una política común. ${ }^{9}$ Sin embargo, aún existen diferencias notables de un país a otro en cuanto a la capacidad para realizar, utilizar y organizar la investigación para la salud, o para dar seguimiento a su capacidad de investigación. A menudo, el rendimiento de la inversión en los planos económico y del desarrollo se observa fuera del ámbito de la salud, en otros sectores del gobierno, la sociedad y la economía. ${ }^{10}$ Algunos ejemplos son la eliminación de la poliomielitis y el acceso al tratamiento de la infección por el $\mathrm{VIH} /$ sida, que permitieron que cientos de miles de personas tengan una vida más plena, sean más productivas y

\footnotetext{
Directora. Organización Panamericana de la Salud/Organización Mundial de la Salud (OPS/ OMS), Washington, D.C., Estados Unidos.
}

brinden una contribución más amplia a la sociedad. ${ }^{11,12}$

En algunos lugares, la organización y la gestión de la investigación es inexistente, y aún no se aprovechan los beneficios de los macrodatos, las redes sociales y otras herramientas digitales. La sociedad aún no ha visto que los beneficios de la era digital se reflejen en el seguimiento y la organización de la investigación para la salud. Sin embargo, las estructuras y los procesos que brindan un mejor apoyo a la realización de la investigación y a las investigaciones sobre los sistemas de salud y la salud pública están listos para ser aprovechados por la comunidad de investigación. La sociedad, los organismos de financiamiento, los patrocinadores y los participantes de las investigaciones podrán entonces ver los resultados y los productos de las inversiones regionales en el campo de la investigación.

Lamentablemente, en la Región de las Américas la investigación sigue siendo una función débil de la salud pública, y eso es algo que debe cambiar. ${ }^{13}$ En el último decenio, las normas relativas a la presentación de información de las investigaciones han mejorado y los registros de ensayos han aumentado la transparencia en el campo de la investigación, pero persiste la necesidad de integrar los avances de manera que beneficien a las personas y de que esto se haga sistemáticamente en todos los países. Detectar las brechas que subsisten ayudará a la Región a enfrentar y eliminar las enfermedades establecidas y emergentes, y a mantener los logros en

\footnotetext{
Editor ejecutivo. The BMJ, Londres, Reino Unido Asesor principal Investigación sobre Sistemas de Salud, OPS/OMS, Washington, D.C., Estados Unidos $\bowtie$ cuervolu@paho.org
} 
Recuadro 1. Objetivos de la política de la OPS sobre investigación para la salud

- Promover la generación de investigaciones pertinentes, éticas y de calidad.

- Fortalecer la gobernanza de la investigación y promover la definición de temarios para la investigación.

- Mejorar la competencia de las personas que participan en la investigación y prestarles un mayor apoyo.

- Promover una mayor eficiencia e intensificar las repercusiones de la investigación y la identificación con sus objetivos mediante alianzas eficaces y estratégicas, la colaboración, el fortalecimiento de la confianza y la participación del público en la investigación.

- Fomentar prácticas y estándares óptimos para la investigación.

- Promover la difusión y utilización de los resultados de la investigación.

cuanto a la salud, la equidad y el acceso. La planificación de las inversiones debe mejorar para que los descubrimientos se traduzcan en logros para la salud pública y los sistemas de salud. ${ }^{14}$

En esta serie de artículos se evalúa el progreso alcanzado desde que se introdujo la política de investigación para la salud de la OPS, se indican las áreas en las que el progreso ha sido lento y se abordan los retos de cara al futuro. Además, agradeceremos los aportes que se sumen al debate en la Región sobre la manera de integrar la investigación a los sistemas de salud, organizar la investigación y aumentar su valor. ¿Cómo debemos configurar los equipos de investigación en un mundo interconectado y cómo hacemos para aprovechar cada oportunidad que pueda surgir?

En la política de investigación de la OPS se adoptó un enfoque sistémico con seis objetivos interrelacionados (véase el recuadro 1); la investigación es necesaria para cumplir las funciones básicas de la OPS y la OMS. ${ }^{215}$ Nuestro propósito es configurar la agenda de investigación en la Región de las Américas y en el mundo al fomentar la generación, la traducción y la difusión de conocimientos de gran valor. Instamos a otras regiones a que transmitan sus experiencias y ayuden a crear una narrativa común para mejorar los sistemas de salud y lograr un mundo más saludable.

\section{REFERENCIAS}

1. Chalmers I, Bracken MB, Djulbegovic B, et al. How to increase value and reduce waste when research priorities are set. Lancet 2014;383:156-65. 10.1016/S01406736(13)62229-1 24411644

2. Cochrane AL. Effectiveness and efficiency: random reflections on health services. Nuffield Provincial Hospitals Trust, 1972.

3. Smith R, RennieD. Evidencebased medicine —an oral history. JAMA 2014;311:365-7. 10.1001/jama.2013.286182 24449049

4. Organización Mundial de la Salud. Llamamiento a la acción de Bamako a favor de las investigaciones en pro de la salud: Fortalecimiento de las investigaciones en pro de la salud, el desarrollo y la equidad. Fortalecimiento de la investigación para la salud, el desarrollo y la equidad. OMS, 2008.

5. Organización Mundial de la Salud. Bamako call to action urges new approach to research for health. 2008. http://www .who.int/rpc/news/bamako_call_to action/en/

6. Organización Panamericana de la Salud. Política de investigación para la salud. Documento CD49/10. 2009. https:/ /www .paho.org/hq/images/stories/KBR/Res earch/politica $\% 20$ de $\% 20$ investigacion $\% 20$ para $\% 20 l a \% 20$ salud.pdf?ua $=1$
7. Organización Mundial de la Salud. 63. ${ }^{a}$ Asamblea Mundial de la Salud. Función y responsabilidades de la OMS en las investigaciones sanitarias. Documento A63/22. 2010. http://www.who.int/gb/ebwha /pdf_files/WHA63/A63_22-sp.pdf

8. Organización Mundial de la Salud. 13. ${ }^{\circ}$ Promover la salud, preservar la seguridad mundial y servir a las poblaciones vulnerables. 2018. http://www.who.int/about /what-we-do/gpw-thirteen-consultation /en/

9. Cuervo LG, Bermúdez-Tamayo C. Desarrollo de la investigación para la salud en Latinoamérica y el Caribe. Colaboración, publicación y aplicación del conocimiento. Gac Sanit 2018;32:206-8. 10.1016/j.gace ta.2018.03.001 29728253

10. Greenhalgh T, Raftery J, Hanney S, Glover M. Research impact: a narrative review. BMC Med 2016;14:78. 10.1186/s12916-016 $-0620-827211576$

11. Thirumurthy H, Galárraga O, Larson B, Rosen S. HIV treatment produces economic returns through increased work and education, and warrants continued US support. Health Aff (Millwood) 2012;31:1470-7. 10.1377/hlthaff.2012.0217 .22778336 Programa General de Trabajo, 2019-2023:
Agradecimientos. A Francisco Becerra, Luis Alejandro Salicrup y el Comité Asesor de Investigaciones en Salud de la OPS por su apoyo en la elaboración de esta serie.

Conflicto de intereses. Hemos leído y comprendido la política de The BMJ sobre declaración de intereses y no tenemos intereses pertinentes que declarar.

Procedencia del artículo y revisión por pares. Comisionado; no hubo revisión externa por pares.

Serie Fortalecimiento de la investigación para la salud en las Américas. Este artículo es parte de una serie propuesta por la Organización Panamericana de la Salud (OPS) y comisionada por The BMJ, que tuvo a cargo la revisión por pares y la edición, y tomó la decisión sobre la publicación de los artículos sin participación de la OPS. Los gastos de publicación en acceso abierto de la serie original en inglés fueron financiados por la OPS. Acceso a la serie original en inglés en www.bmj.com /health-research-americas; accesoalaserie en español y portugués en https://www .paho.org/journal/es/numeros-espec iales / fortalecimiento-investigacion-para -salud-americas.
12. Zandam H. Life after polio: towards improving the situation of polio survivors. 2015. https://www.twigh.org /twigh-blog-archives/2015/8/25/life -after-polio-towards-improving-the -situation-of-polio-survivors

13. Organización Panamericana de la Salud. Public health in the Americas: conceptual renewal, performance assessment, and bases for action. 2002. http:/ / iris.paho.org/ xmlui/handle/123456789/2748

14. Organización Mundial de la Salud. Changing mindsets-strategy on health policy and systems research. 2012. http:/ /www .who.int/alliance-hpsr/alliancehpsr_chan gingmindsets_strategyhpsr.pdf

15. Organización Panamericana de la Salud. Valores, misión y visión de la Organización Panamericana de la Salud. 2015. https:// www.paho.org/hq/index.php?option= com_content\&view $=$ article $\& i d=91$ : about-paho\&Itemid=220\&lang=es.

Forma de citar (artículo original): BMJ 2018;362: k2944 doi: http:/ /dx.doi.org/10.1136/bmj.k2944

Manuscrito original en inglés publicado el 16 de julio de 2018. 